\title{
Respecto a "Guías para la Monitorización \\ Ambulatoria de Presión arterial"
}

Hernán Prat

Hospital Clínico Universidad de Chile.

Sr. Editor:

Concomitante con el procesamiento editorial de las "Guías para la monitorización ambulatoria de presión arterial de 24 horas" en Rev Chil Cardiol 2017; 36: 264-274', apareció, a mediados de noviembre pasado la publicación "Guías para la prevención, detección, evaluación y manejo de la hipertensión en adultos" en el Journal of the American College of Cardiology (JACC) ${ }^{2}$ por Welthon P, et al. En estas guías se ponen otros umbrales para hipertensión arterial y se extrapolan para valores del monitoreo ambulatorio de presión arterial (MAPA).

$\mathrm{Al}$ respecto y sin ánimo de criticar esas guías, podemos decir que: 1) esta nueva publicación del JACC no ha sido avalada hasta el momento por Sociedades Europeas, Cana- diense ni la del Ministerio de Salud de Chile; 2) La extrapolación de valores que se ponen en esas guías (cuadro 11) siguen a valores propuestos por los autores, descontando $5 \mathrm{~mm} \mathrm{Hg}$ para MAPA. Este descuento se hizo hace años para compensar cifras obtenidas con manómetros de mercurio, situación que en la actualidad es discutible, ya que prácticamente se ha descartado el uso de esos equipos. 3) No hay evidencias en la literatura que los valores propuestos para MAPA impliquen menor morbimortalidad que los que nosotros publicamos.

Por tanto estimamos que, hasta que no se aclaren y avalen las normas publicadas por Welthon y cols., las guías queden como las hemos publicado y, si hubiera que hacer modificaciones, las haremos llegar oportunamente a la Revista Chilena de Cardiología. 
1. HERNÁN PRAT, ALEJANDRO ABUFHELE, GONZALO ALARCÓN, INÉS BARQUÍN, EDGARDO ESCOBAR, MAURICIO FERNÁNDEZ, et al. Guías para la monitorización ambulatoria de presión arterial de 24 horas. Documento de la Sociedad Chilena de Cardiología y Cirugía Cardiovascular. Rev Chil Cardiol 2017; 36: 264-274.
2. WHELTON PK, CAREY RM, ARONOW WS, CASEY DE JR, COLLINS KJ, DENNISON HIMMELFARB C, et al. 2017 ACC/AHA/AAPA/ABC/ACPM/AGS/APhA/ASH/ASPC/ NMA/PCNA Guideline for the Prevention, Detection, Evaluation, and Management of High Blood Pressure in Adults: A Report of the American College of Cardiology/American Heart Association Task Force on Clinical Practice Guidelines. J Am Coll Cardiol. 2017; 70: 735-1097. 